EDITORIAL

\title{
Preventing recurrent rheumatic fever: the role of register based programmes
}

\author{
M McDonald, A Brown, S Noonan, J R Carapetis
}

Heart 2005;91:1131-1133. doi: 10.1136/hrt.2004.057570

Despite dramatic gains of the last century, acute rheumatic fever and rheumatic heart disease remain major preventable causes of morbidity and mortality in many parts of the world

See end of article for authors' affiliations

.....................

Correspondence to: Dr Malcolm McDonald Menzies School of Health Research, PO Box 41096 Casuarina, Northern Territory, 0811, Australia; malcolm@menzies.edu.au
A cute rheumatic fever (ARF) and rheumatic heart disease (RHD) have all but disappeared from countries with industrialised market economies. Yet the global burden of disease remains substantial; a minimum of 15.6 million people are estimated to have established RHD, and close to 300000 new cases of RHD are identified each year with 233000 directly attributable deaths (JR Carapetis, unpublished report for the World Health Organization). The predominant risk factor is poverty.

Antibiotic treatment of streptococcal pharyngitis (primary prophylaxis) can prevent ARF in well resourced settings but is less practical where disease rates are high. ${ }^{1}$ It is also unlikely that an effective and affordable vaccine will be available in the near future. Secondary prevention requires diagnosis of ARF/RHD followed by long term antibiotic prophylaxis. It is the only strategy proven to be cost effective but ideally requires the support of a register based programme. Tertiary prevention-medical and surgical treatment of people with established RHD-is the least cost effective approach.

Register based ARF/RHD secondary prevention programmes were established across the USA in the 1950s when national rates of disease were already falling rapidly. The contribution these programmes played in subsequent decline is unclear, ${ }^{2}$ but, overall, they were considered to be a successful long term strategy. ${ }^{3}$ National guidelines were published for secondary prevention and establishment of ARF/RHD registers in $1970 .{ }^{4}$ By 1981, only 11 states reported active programmes. ${ }^{5}$ Today, neither the Centers for Disease Control and Prevention nor the American Heart Association maintains records of programmes that are operational.

\section{WHO REGISTER BASED PROJECT}

A register based project for control of ARF/RHD was launched by the World Health Health Organization (WHO) in 1972. ${ }^{6}$ Although surveillance was incomplete and reported follow up rates were around 50\%, the estimated reduction in subsequent health costs was considerable. WHO then embarked upon a Global Programme and, by 1990, ARF/RHD registers had been established in 16 counties, with almost 1.5 million school age children screened and over 3000 cases of RHD or prior ARF detected. ${ }^{7}$ A later review highlighted improved compliance with secondary prophylaxis. ${ }^{8}$ Subsequently, only a handful of countries expanded their programmes and funding for the WHO Global Programme ceased in 2001. It is not clear how many countries continue programmes with internal funding.

The New Zealand experience provides compelling evidence in favour of register based programmes. Most were developed in the 1970s with notable successes in reducing ARF recurrences. ${ }^{9}$ In 1986, ARF became a notifiable disease but attempts to set up a national register failed because of privacy concerns. By 1998, half the country's health districts had ARF/RHD registers, covering 94\% of notified ARF cases. Successful register based programmes in India, Cuba, and Egypt have demonstrated how they can be implemented at low cost using existing infrastructure. ${ }^{10}{ }^{11}$ Programmes have also recently been established in Australia to serve Aboriginal populations. ${ }^{12} 13$

Nevertheless, at the start of the new century, there is no comprehensive international strategy and there are few active national programmes. ARF and RHD are potentially preventable conditions but will remain unchecked among the world's poorest populations unless current prevention programmes expand and new programmes are established.

\section{VALUABLE EPIDEMIOLOGICAL TOOL}

An accurate and up-to-date register of people with known past ARF and RHD is critical for efficient delivery of prophylaxis and to monitor service delivery. It is also a valuable epidemiological tool. Establishing and maintaining a register based prevention programme is a complex and relatively expensive process. ${ }^{14}$ Various models exist, ranging from actively interventional to simply providing information for independent health practitioners to act upon. They may be integrated into a broad public health strategy or purpose specific and stand alone. They may be hospital based, practitioner based, or community based.

Registers require careful planning. Choice of software is based on cost, availability, local computer expertise, and potential for integration with other health information systems, such as hospital databases. Registers should be kept

Abbreviations: ARF, acute rheumatic fever; RHD, rheumatic heart disease; WHO, World Health Organization 
Table 1 Acute rheumatic fever/rheumatic heart disease (ARF/RHD) register: example of priority based guidelines*

Priority 1: severe disease

Established RHD with any of

- A valvar lesion that is severe

- A moderate to severe valvar lesion where left ventricular (LV) function is impaired or LV size is increased

- A moderate to severe valvar lesion where there is shortness of breath, tiredness, oedema, angina, or syncope

- History of bioprosthetic valve replacement (porcine or homograft), valve repair, valvotomy or metallic prosthetic valve replacement (until stabilised)

Management:

- Management by a cardiologist and consideration for surgery

- Patients should be reviewed every 6 months by a specialist physician or paediatrician

Priority 2: moderate disease

Established RHD with any of:

- A valvar lesion that is moderate, providing there are no symptoms and left ventricular function is normal

- Mechanical prosthetic valves once stable following surgery

- A child or adolescent with a history of chorea until 18 years old, even if there is no valve damage ( $>50 \%$ will subsequently develop valve disease)

Management:

- Managed by primary care doctor with review by specialist physician/ paediatrician every year (or earlier if clinical deterioration) or within 3 months of hospital discharge following any episode of confirmed or suspected ARF

- Echocardiogram recommended every year (children) or 2 years (adults) to assess valve lesion severity and LV function

Priority 3: mild disease

RHD or ARF

- A valvar lesion that is trivial to mild

- History of ARF with no evidence of RHD

Management:

- Managed by primary care doctor unless clinical deterioration. Children and adolescent up to 18 years reviewed every year by a specialist physician or paediatrician

- Echocardiogram every 2 years (children) or 5 years (adults, no recent ARF).

- Any new diagnosis of ARF always requires specialist physician/ paediatrician follow up within 3 months of hospital discharge to assess progress

- Specialist physician review before ceasing secondary prophylaxis

*Adapted from the Northern Territory Rheumatic Heart Disease Register 2003, with permission.

up-to-date with reliable systems to disseminate information and mechanisms to identify and locate patients lost to follow up. Accurate reporting requires data integrity without duplications and contradictions. To optimise use of limited resources, a priority system can be used that indicates severity of disease and identifies individuals at particular risk (table 1). Reliable sources of information will include hospital discharge and clinic records, echocardiogram and laboratory reports, official notifications, and medical correspondence.

Understanding local context is critical. Communities with the highest rates of ARF/RHD are often the least equipped to mount a response. Language and religious diversity, levels of education, concepts of sickness and health, community tensions, and population mobility must be considered. Initial consultation with local health providers will identify available resources together with potential structural and social barriers to implementation. It will also instil a sense of community ownership.

\section{STEERING COMMITTEE}

Most programmes require a steering committee and a coordinator. The steering committee plans the structure of the programme and meets regularly to review progress. It should provide recommendations about management, seek external advice where necessary, and secure funding. The committee should include people with a wide range of interests and skills who have influence at local, regional, and national levels. The programme coordinator ideally should have a nursing or other health related background, with computer competency and self direction. The role of the coordinator includes supervising care across health services, identifying people with known or suspected ARF/RHD, maintaining the register and producing regular reports, developing local strategies to improve adherence to secondary prophylaxis, diagnosis and management, educating patients, families and key health staff about ARF/RHD, and evaluating success of programme activities.

Potential problems include under reporting of cases, requirements for informed consent, and privacy. Under reporting compromises the reliability of the register..$^{15} \mathrm{~A}$ semi-automated system, in which patients admitted to hospital with a possible diagnosis of ARF or RHD are routinely identified, may be useful. We have also found it helpful to have ARF made notifiable by the Ministry of Health, so that medical staff are bound by law to notify cases to health authorities.

\section{INFORMED CONSENT}

Informed consent is often required for patients to be on a disease register. This can be a formidable and expensive undertaking, especially for registers established before the informed consent process. Moreover, in settings with the highest rates of ARF/RHD, educational services are often limited, cultural differences may be substantial, and there may be suspicion of the consent process. The process itself may even pose a barrier to enrolment and threaten survival of the programme. ${ }^{16}$ It is essential to keep the consent process simple and make the public aware of the register's benefits. Privacy legislation can pose a barrier to effective use of register information. In many settings, access to data for research purposes is only available when considered to be in the overriding public interest. This is often difficult to establish. The implications of privacy legislation and consent require careful consideration before programmes are established.

Despite dramatic gains of the last century, ARF and RHD remain major preventable causes of morbidity and mortality. Secondary prevention, through prophylactic antibiotics and register based programmes, is the most efficient and cost effective intervention. There is no better time to consider reestablishing programmes, especially where the burden of disease is highest. This requires long term support from international bodies such as the WHO, governments, health professionals and local communities, with provision of adequate and sustainable funding.

\section{Authors' affiliations}

M McDonald, Menzies School of Health Research, Charles Darwin University, Darwin, Australia

A Brown, Alice Springs Campus of the Menzies School of Health Research, Charles Darwin University, Alice Springs, Australia

S Noonan, Department of Thoracic Medicine, Royal Adelaide Hospital, Adelaide, Australia

J Carapetis, Centre for International Child Health, University of Melbourne, Royal Children's Hospital and Murdoch Children's Research Institute, Melbourne, Australia

Competing interests: Malcolm McDonald, Alex Brown and Jonathan Carapetis are supported by grants from the National Heart Foundation (Australia) and the National Health and Medical Research Council. Jonathan Carapetis has grants from a number of other sources including the National Institutes of Health (NIH, USA) and has acted as a consultant on rheumatic heart disease matters for both NIH and WHO. Otherwise the authors have no conflict of interest. 


\section{REFERENCES}

1 World Health Organization. Strategy for controlling rheumatic fever/ rheumatic heart disease, with emphasis on primary prevention: memorandum from a joint WHO/ISFC meeting. Bull World Health Organ 1995;73:583-7.

2 Quinn R. Comprehensive review of morbidity and mortality trends for rheumatic fever, streptococcal disease, and scarlet fever: the decline of rheumatic fever. Rev Infect Dis 1989;1 1:928-53.

3 Tompkins D, Boxerbaum B, Liebman J. Long-term prognosis of rheumatic fever patients receiving regular intramuscular benzathine penicillin. Circulation 1972;45:543-51.

4 Taranta A, Fielder J, Frank C, et al. Prevention of rheumatic fever and rheumatic heart disease. Circulation 1970;61:A1-15.

5 Kaplan EL. Current status of rheumatic fever control programs in the United States. Public Health Rep 1981;96:267-8.

6 Strasser T, Dondog N, El Kholy A, et al. The community control of rheumatic fever and rheumatic heart disease: report of a WHO international cooperative project. Bull World Health Organ 1981;59:285-94.

7 World Health Organization. WHO programme for the prevention of rheumatic fever/rheumatic heart disease in 16 developing countries: report from phase 1 (1986-90). Bull World Health Organ 1992;7:213-18.
8 World Health Organization. The WHO global programme for the prevention of rheumatic fever and rheumatic heart disease: report of a consultation to review progress and develop future activities. Geneva: WHO, 2000:1-49.

9 Thornley C, McNicholas A, Baker M, et al. Rheumatic fever registers in New Zealand. NZ Pub Health Rep $2001 ; 8(6): 41-4$.

10 Kumar R, Raizada A, Aggarwal AK, et al. A community-based rheumatic fever/rheumatic heart disease cohort: twelve-year experience. Indian Heart J 2002;54:54-8

11 World Health Organization. Rheumatic fever and rheumatic heart disease: report of a WHO expert consultation. Geneva: WHO 2004.

12 Noonan S, Edmond K, Krause V, et al. The top end rheumatic heart disease control program I. Report on progress. NT Dis Control Bull 2001;8:15-18.

13 Brown A, Purton L, Schaeffer G, et al. Central Australian rheumatic heart disease control program: a report to the Commonwealth, November 2002. NT Dis Control Bull 2003;10:1-8.

14 Solomon DJ, Henry RC, Hogan JG, et al. Evaluation and implementation of public health registries. Public Health Rep 1991;106:142-50.

15 Rice MJ, Kaplan EL. Rheumatic fever in Minnesota. II. Evaluation of hospitalized patients and utilization of a state rheumatic fever registry. Am J Public Health 1979:69:767-71.

16 Verity C, Nicoll A. Consent, confidentiality, and the threat to public health surveillance. BMJ 2002;324:1210-12.

\section{IMAGES IN CARDIOLOGY}

\section{Rare case of pericarditis constrictiva}

A 68 year old man was admitted to the emergency room with recurrent syncopal attacks and clinical signs for a distinct right heart insufficiency. Clinical history was marked by a mild hepatopathy after hepatitis B and recurrent pleuritis treated by talc pleurodesis. No bacterial infection or systemic inflammation was detected. After the exclusion of neurological pathologies cardiac examination showed normocardiac atrial fibrillation and a left anterior hemi-block. Due to massive pericardial calcifications transthoracic echocardiography revealed only low quality images and was not conclusive. Left heart catheterisation confirmed a normal sized left ventricle showing the classical plateau phenomenon with equalisation of end diastolic pressure (panel A).

Chest $x$ ray showed an increased heart size with impressive pericardial calcifications. We also found pleural densities in the right lower lung with mild effusions of the right pleural cavity, calcifications on the left basal area, and a pulmonaryvenous congestion (panel B). Coronary angiography depicted three vessel disease with significant stenoses of the left anterior descending artery (LAD), circumflex artery (CX), and right coronary artery (RCA). Intraoperative exploration confirmed extensive pericardial calcifications. Because of the

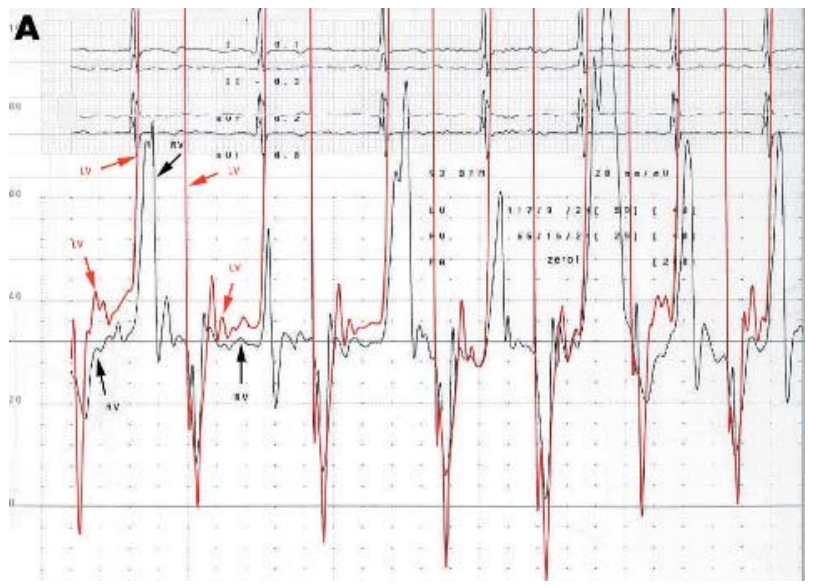

doi: $10.1136 /$ hrt. 2004.056820

$2 \mathrm{~cm}$ thick calcified layer the pericardium could only be opened by an oscillating saw and surgical removal of the calcifications was extremely difficult. Concerning the increased cardiac stiffness, luxation for a postero-basal bypass to the $\mathrm{CX}$ proved to be too dangerous and only bypasses to the LAD and RCA were performed. Clinical follow up was uneventful with restored cardiac function and rapid improvement of right ventricular function. The origin of these impressive pericardial calcifications remains unclear.
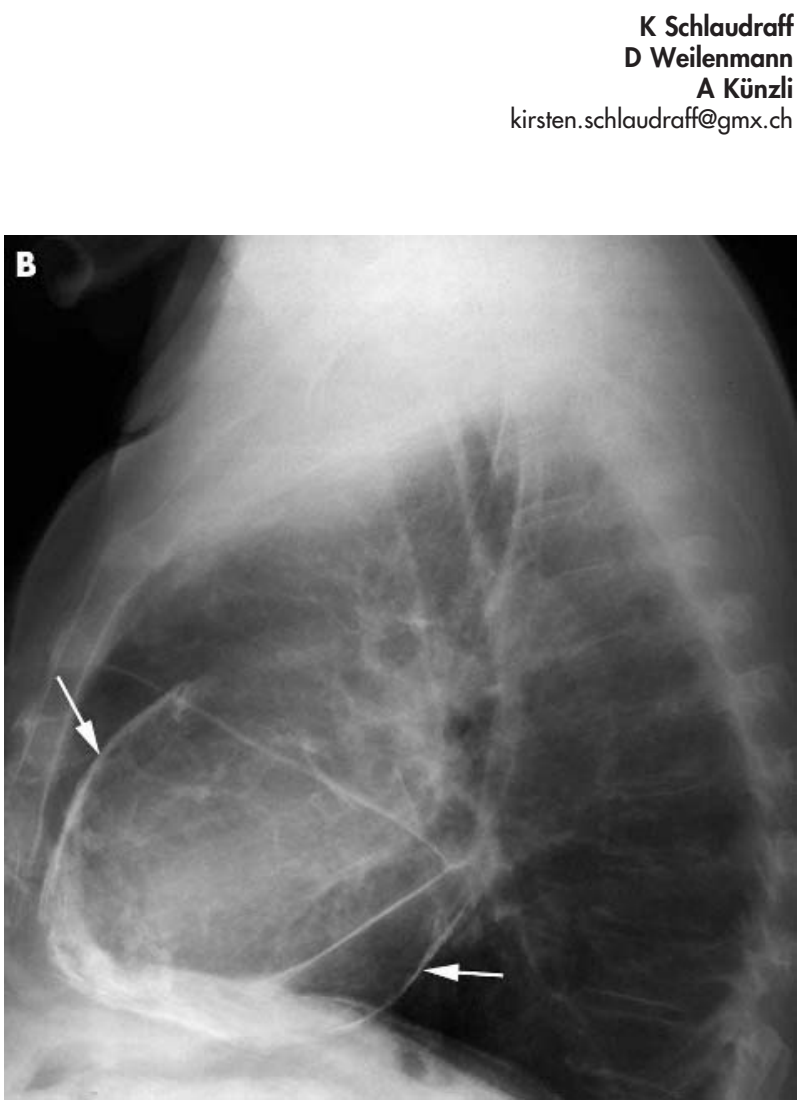